\title{
An Architecture for Simulating Needle-Based Surgical Procedures
}

\author{
Alan Liu, Christoph Kaufmann, Daigo Tanaka \\ \{aliu|ckaufmann|dtanaka@simcen.usuhs.mil\} \\ National Capital Area Medical Simulation Center \\ Uniformed Services University of the Health Sciences \\ 4301 Jones Bridge Road, Bethesda MD 20814, USA \\ http://simcen.usuhs.mil
}

\begin{abstract}
Many surgical procedures use cannulas, guidewires, and catheters in the treatment of life threatening conditions (e.g. cardiac tamponade and tension pneumothorax), or for diagnosis (e.g. diagnostic peritoneal lavage). Simulator development is costly in time and resources. Most computer-based trainers are procedure-specific. Each trainer uses a different hardware configuration. The cost of using multiple simulators for teaching is prohibitive. A result is decreased acceptance of simulation for teaching. A generalized software architecture has been developed that simplifies the process of constructing trainers for needle-based surgical procedures. Different procedures can use the same hardware platform. The architecture has been used to develop two trauma simulators. A third simulator is currently being developed using this architecture.
\end{abstract}

\section{Introduction}

Computer-based trainers have been developed for teaching many surgical procedures [1-4]. Simulator-based training has many advantages. Unlike cadavers or anesthetized animals, simulators require minimal setup and preparation. They are reusable, and provide a uniform training experience. Unlike animals, simulators present the student with the correct human anatomy. Simulators can also provide the appropriate physiological responses, unlike cadavers.

Despite these advantages, the acceptance of computer-based simulators for medical education has been weak. One reason is cost. Many simulators require a custom hardware configuration. The hardware may not be usable for a different procedure. Since each simulator is unique, their development and production cost is high. A course involving many different procedures will require several expensive systems. For many institutions, the expense cannot be justified.

This paper describes an architecture for developing trainers for needle-based procedures. Many procedures fall under this category. They include: Diagnostic peritoneal lavage (DPL), pericardiocentesis, needle thoracentesis, needle cricothyroidotomy, central line placement, intraosseous puncture, and needle biopsies of the thyroid and breast. An architecture using a common hardware platform and development approach for a class of procedures addresses the problems raised earlier. 
In this paper, section 2 describes the hardware and the architecture. Section 3 describes simulators constructed using this architecture. Section 4 describes the implications of this work.

\section{Method}

The architecture is based on the premise that a surgical procedure can be broken down into a series of discrete steps. The user is assumed to be familiar with the procedure's algorithm. A successful outcome requires the user to complete a sequence of steps correctly. Other outcomes with varying degrees of success are possible, depending on the user's actions and the number of errors made during the procedure.

Finite automata [5] provide a natural way of representing surgical algorithms. A finite automata consists of a set of states and transitions between states. Each state represents a specific action to be completed. The student's performance determines the transition between states. For example, when a cannula is incorrectly inserted, a transition back to the current state permits the student to try again. A correct insertion invokes a transition to a different state to perform the next step.

The architecture consists of several components: A state engine, a graphical user interface, a haptic interface, and a transition algorithm. Each is described in more detail.

\subsection{State Engine}

The state engine is the architecture's core. The engine incorporates a finite automata (FA), and a state transition algorithm. This algorithm is described in detail in section 2.4. The FA consists of a set of states, and a state transition table. Each state represents a step in a surgical procedure. The user interacts with state objects to perform the corresponding surgical action. A simulation consists of executing a series of steps. As the student performs each step, his performance together with the transition table will be used to determine the subsequent step.

Each state is defined by three functions. The init() function initializes the state. The run() function executes the surgical step. get_result() reports the result of that execution. Depending on the procedure, different states have different definitions for each function. A state may be distinguished as a start or an end state. The simulation will always begin on a start state. End states denote that the simulation has completed. An end state is characterized by having no other states to which that state can perform a transition. Depending on the student's actions, end states may denote a successful or unsuccessful completion. There is exactly one start state at least one end state.

\subsection{Graphical User Interface}

The GUI object consists of sequence window and an action window. Fig. 1 illustrates. The sequence window presents a randomized list of steps that must be performed in the procedure. The action window provides visual feedback on actions performed by the student during each step of the procedure. During a simulation, the user selects the current step using the sequence window. If the correct step is chosen, the action 
window becomes active and prompts the user to perform the chosen step (e.g., inserting a cannula). The user's actions are displayed in the action window. Successful completion of the current step re-activates the sequence window where the next step can be chosen.
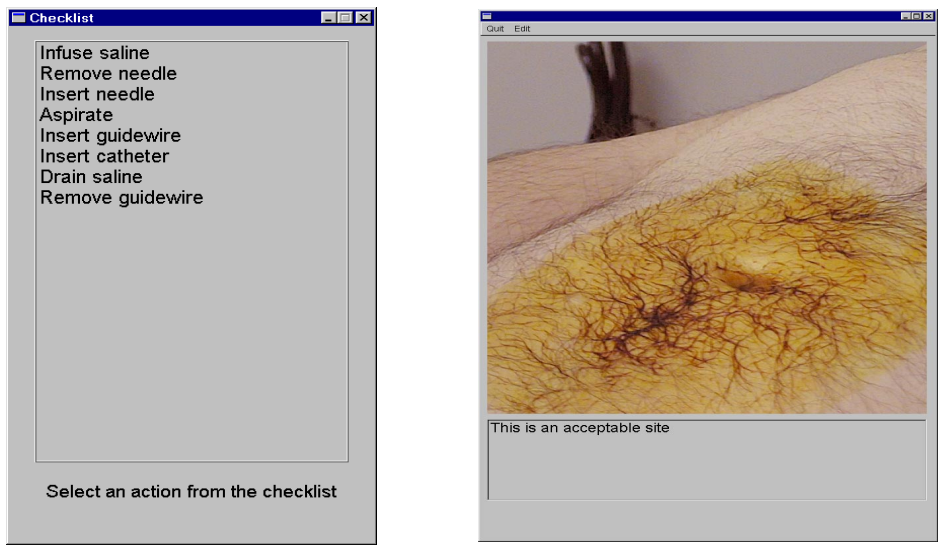

Fig. 1. GUI interface as configured for diagnostic peritoneal lavage.

\subsection{Haptic Interface}

The CathSim AccuTouch ${ }^{\circledR}$ device [6] by Immersion Medical (formerly HT Medical) is employed. The haptic interface consists of an enclosed needle carrier. Sensors on the needle carrier report pitch, yaw, and depth. The needle carrier accepts cannulas, catheters and guidewires. Encoders on the carrier can detect the depth to which a cannula or guidewire has been inserted. Up to two instruments (e.g., a cannula and a guidewire) can be simultaneously inserted and tracked. The needle carrier incorporates passive haptics in the depth direction. As the carrier is pushed or pulled, a variable amount of braking force can be applied. By dynamically controlling resistance, the sensation of a cannula penetrating various tissue layers can be accurately simulated. Fig. 2 illustrates.

\subsection{Execution and Transition Algorithm}

The sequence of states executed by the simulation is determined by this algorithm.

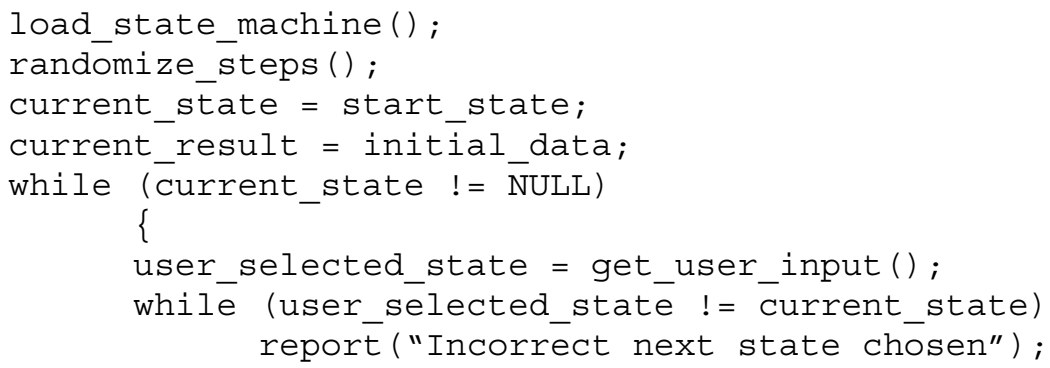




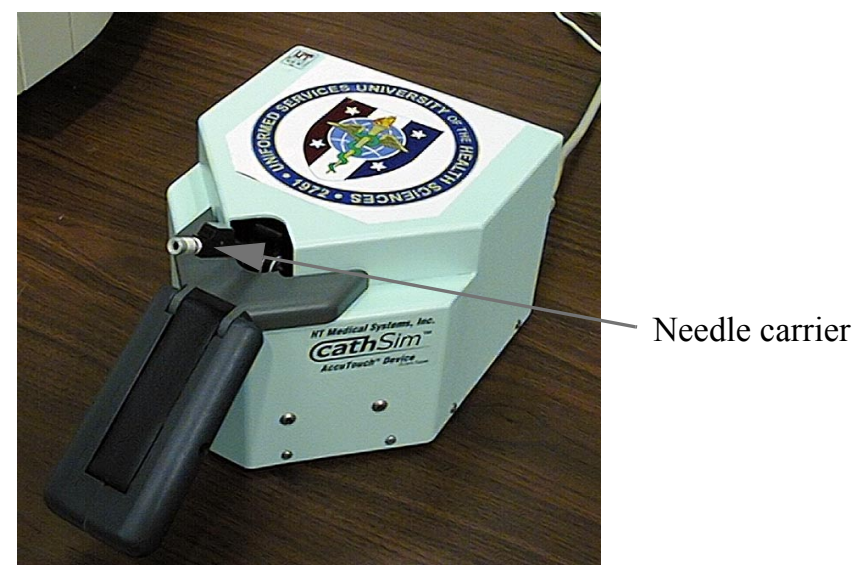

Fig. 2. Haptic interface device.

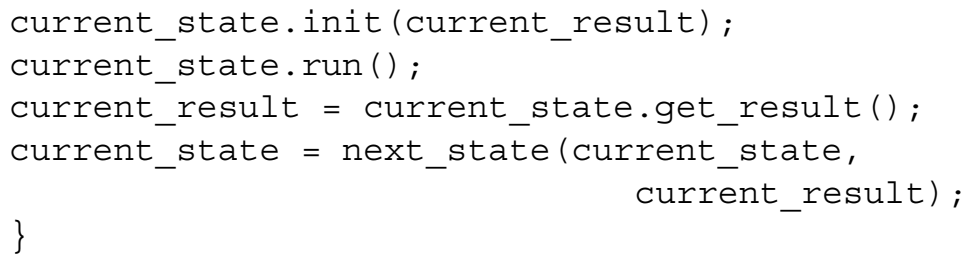

The simulator begins by loading the state machine defining the procedure to be performed. The procedure's steps are randomized prior to display. The simulator is initialized with the start state and data from initial_data. The user is prompted to select the first step of the procedure. The function get_user_input() displays the sequence window and prompts the user to click on the correct step to perform. If an incorrect choice is made, an error message is displayed. When the correct state is chosen, that state is initialized (current_state.init()), then run (current_state.run()). The result of this execution is returned (current_state.get_result()) and stored in current_result. The next state executed is determined by the current state (current_state), and by the results in current_result. The results are also used to initialize the next state. This cycle is repeated until an end state is encountered. This is characterized by a state that does not select a new state (i.e., next_state(current_state,current_result) == NULL). Finally, the simulation displays a summary of the student's performance (display_report()).

\section{Case Studies}

This section highlights some of the simulators built using this architecture. At the time of writing, two simulations have been completed. A third simulator is currently being developed. We describe each in turn. 


\subsection{Pericardiocentesis}

Pericardiocentesis is a surgical procedure performed to relieve fluid buildup between the heart and the pericardium. This condition (cardiac tamponade) interferes with the heart's ability to pump blood. Cardiac tamponade must be corrected rapidly, or the patient may die. Initial treatment involves inserting a cannula between the pericardium and heart, then aspirating the blood to relieve pressure buildup. An earlier version based on the Phantom haptic interface device was developed [4]. This simulation has been re-written using the architecture described in this paper. The CathSim device is used in place of the Phantom. Fig. 3 (left) is an image from the GUI.

\subsection{Diagnostic Peritoneal Lavage}

DPL is performed when intra-abdominal bleeding secondary to trauma is suspected. The procedure is performed when alternative diagnostic methods such as computerized tomography (CT) or ultrasound imaging are unavailable, or when the patient's condition does not allow them to be performed. The DPL procedure is complex, involving up to nine steps. Details of this procedure and the simulation have been previously described [3]. Fig. 3 (right) is an image from the GUI. The DPL simulation uses the architecture described in this paper. Fig. 4 illustrates the state machine.
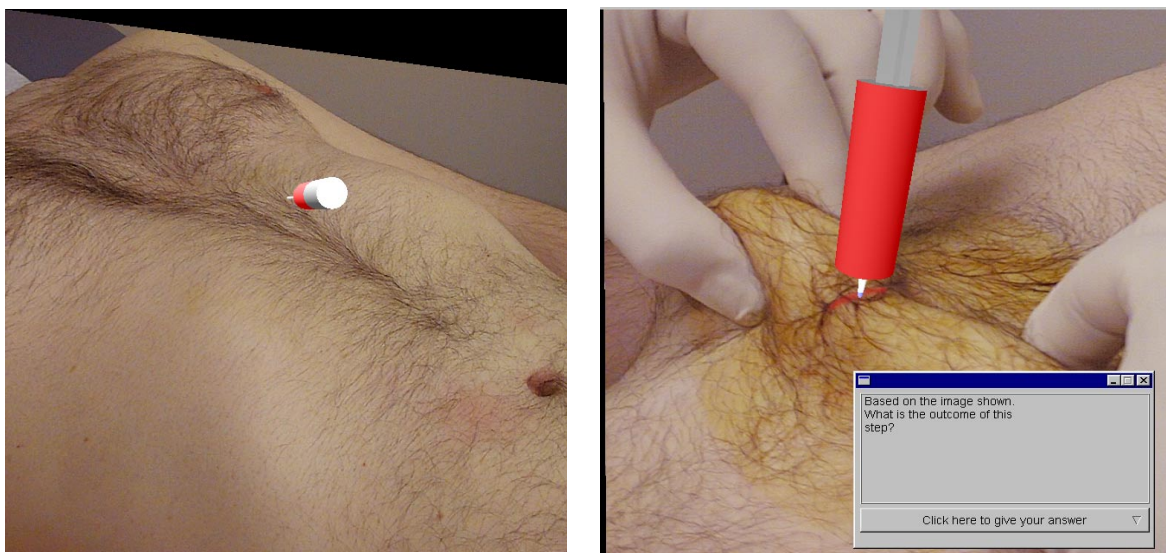

Fig. 3. (left) Pericardiocentesis. (right) Diagnostic peritoneal lavage.

\subsection{Needle Thoracentesis of Tension Pneumothorax}

Needle thoracentesis is used as an immediate treatment for life-threatening tension pneumothorax. This condition can result from chest trauma. Tension pneumothorax occurs when air leaks into the thoracic cavity and cannot escape. The increasing pressure compresses the heart and lungs, causing circulatory and respiratory problems. The procedure requires the insertion of a catheter in the second intercostal space, in the midclavicular line on the side of the tension pneumothorax. The catheter permits excess air pressure to escape. This simulation is currently being developed. The state 


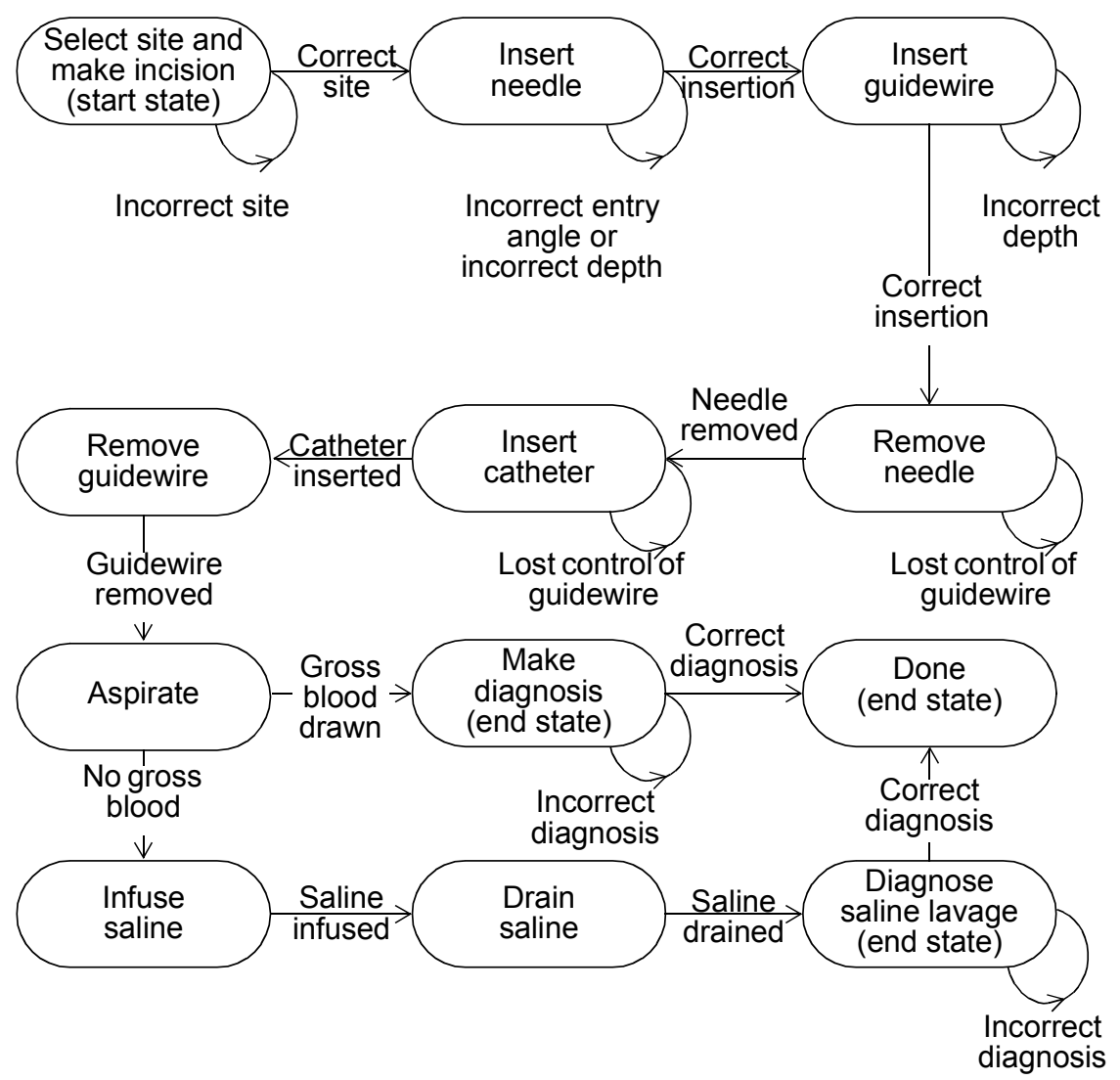

Fig. 4. State machine for diagnostic peritoneal lavage.

machine for this procedure is summarized in fig. 5. This simulation is more elaborate than the previous two in that the consequence of an incorrect action (e.g., incorrect needle insertion), is not apparent until a few steps later. The simulation permits the student to back up and correct the mistake.

\section{Discussion}

Surgical simulators can be an improvement over traditional methods of surgical education. Many simulators have been developed using unique and expensive hardware configurations. The hardware is procedure-specific. Multiple simulator platforms are required for different procedures. The cost of simulating multiple procedures is prohibitive.

Our approach uses a common platform to simulate a large class of procedures. These procedures are commonly taught or used. The chosen hardware platform is 


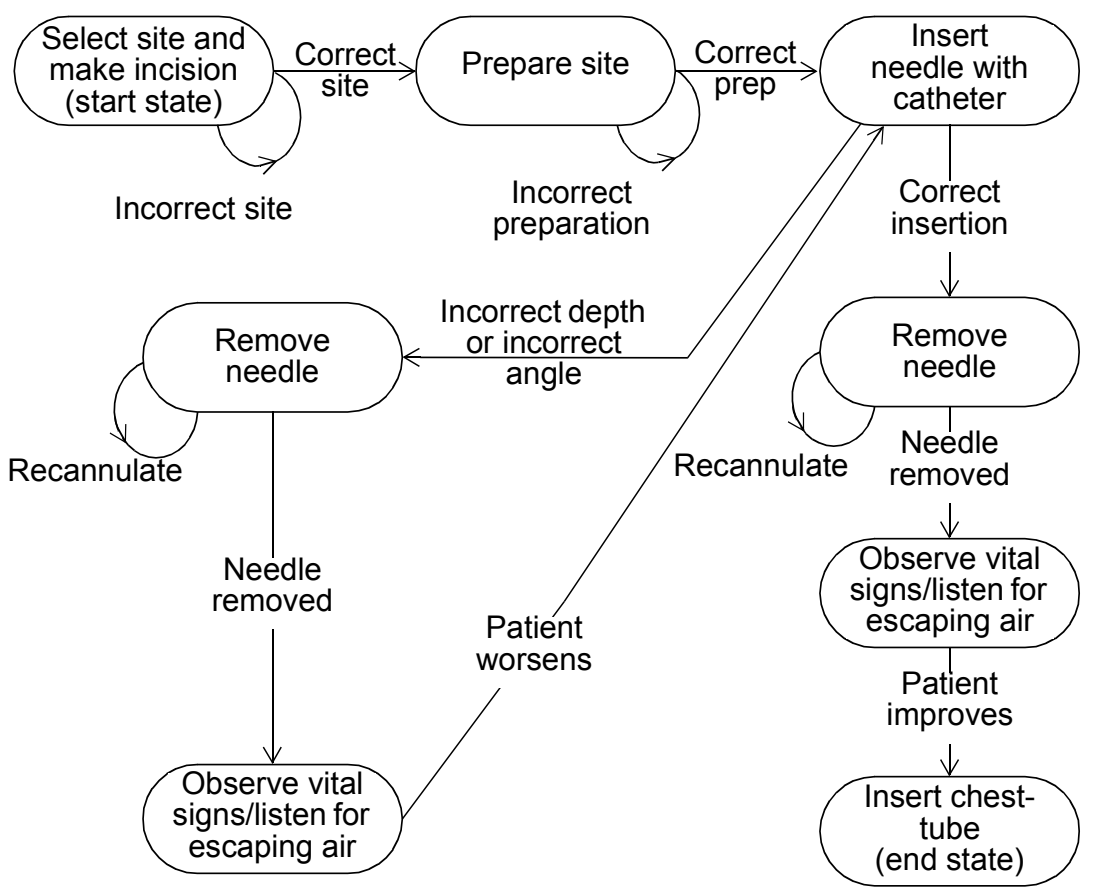

Fig. 5. State machine for needle thoracentesis.

commercially available and is relatively inexpensive. As all simulations built using our design run on the same hardware, no additional hardware investment is required.

Since needle-based procedures have many similarities, the architecture permits many needle-based procedures to be developed with minimal effort. Development effort is focused on defining the individual states, and the transition between states. User interface development, haptic device interface and other peripheral issues need not be considered. Our approach has limitations however. Since only basic hardware is assumed, the degree of realism may be limited. However, in many cases, a high level of realism is not required. Key aspects of learning a procedure include executing the surgical algorithm correctly, using the correct surgical instruments, and managing complications. Our simulation architecture permits these goals to be accomplished. In addition, haptic feedback is primarily limited to one DOF in needle-based procedures. Our choice of haptic interface is sufficient to provide the required feedback.

\section{Conclusion}

We described an architecture for developing needle-based procedures. Our approach is an improvement over conventional methods. Our method permits training simulators to be developed rapidly on a common, low-cost platform. We have validated our architecture by developing two simulations for different needle-based procedures 
using this approach. A third simulation is currently being developed using the same approach.

\section{Acknowledgements}

This work was funded in part by a grant from the Association of Military Surgeons of the United States (AMSUS). The authors gratefully acknowledge the assistance of Immersion Medical Inc. for providing the CathSim AccuTouch device and associated interface. The authors would also like to acknowledge Scott Zakaluzny for his assistance in acquiring the anatomical models used in the simulators described.

\section{References}

1. Piper S., Deph S., Rosen J., Fisher S. "A virtual environment system for simulation of leg surgery." Proc. of Stereoscopic Display and Applications //. SPIE 1991; 1457. pp. 188-196.

2. Meglan D. A., Raju R., Merril G. L., Merril J. R., Nguyen B. H., Swamy S. N., Higgins G. A. "Teleos virtual environment for simulation-based surgical education." Interactive Technology and the New Paradigm for Healthcare, Satava R. M. Morgan K. s., Sieburg H. B., Masttheus R., Christensen J. P., eds. Washington, DC: IOS Press, 1995. pp. 221-230.

3. Alan Liu, Kaufmann C., Ritchie T. "A computer-based simulator for diagnostic peritoneal lavage." Medicine Meets Virtual Reality 2001. Westwood J. D., et al., eds. IOS press, 2001. pp. 279-285.

4. Christoph Kaufmann, Zakaluzny S., Liu A. "First steps in eliminating the need for animals and cadavers in Advanced Trauma Life Support ${ }^{\circledR}$." Medical Image Computing and Computer-Assisted Intervention (MICCAI) 2000. Lecture Notes in Computer Science 1935, Springer. pp. 618-623.

5. Hopcroft J. E., Ullman J. D. "Introduction to automata theory, languages, and computation." Addision Wesley, 1979. ISBN 0-201-02988-X.

6. Ursino, M.; Tasto, P.D.J.L.; Nguyen, B.H.; Cunningham, R.; Merril, G.L. "CathSimTM: an intravascular catheterization simulator on a PC." Medicine Meets Virtual Reality. Convergence of Physical and Informational Technologies: Options for a New Era in Healthcare. MMVR 7, 1999, Netherlands. pp. 360-366 